\title{
Surgical Treatment of Trichilemmal Carcinoma
}

\author{
De Bin $\mathrm{Xu}^{\mathrm{a}, \mathrm{d}}$, Tao Wang ${ }^{\mathrm{b}, \mathrm{d}}$, Zhen Liao ${ }^{\mathrm{c}, \mathrm{e}}$
}

\begin{abstract}
Background: To investigate the clinicopathologic characteristics and prognostic factors of trichilemmal carcinoma (TC), and to determine an optimal treatment strategy for these patients.
\end{abstract}

Methods: This retrospective study enrolled consecutive patients who were admitted to the Sun Yat-sen University Cancer Center between 1998 and 2012.

Results: The key prognostic factors influencing the survival were lymph nodes metastasis and surgery margin. Multivariate analysis revealed that there was no risk factor for patient survival.

Conclusions: Surgery margin and lymph nodes metastasis were prognostic factors that influenced the treatment outcome. Simple excision with $1 \mathrm{~cm}$ margins is safe, inexpensive and effective for the treatment of TC; and postoperative follow-up of the patient to facilitate early diagnosis of the recurrence and distant metastasis is necessary. Systemic chemotherapy should be considered for the distance metastases patients.

Keywords: Trichilemmal carcinoma; Locoregional recurrence; Lymph nodes metastasis; Treatment strategy; Chemotherapy

\section{Introduction}

Trichilemmal carcinoma (TC) is a rare malignant tumor that develops from the external root sheath of the hair follicle. It

Manuscript submitted August 11, 2018, accepted September 6, 2018

${ }^{a}$ Department of Thyroid and Neck Surgery, The Second Affiliated Hospital of Nan Chang University, NanChang, China

bDepartment of Otolaryngology-Head and Neck Surgery, The Third Affiliated Hospital of Sun Yat-sen University, Guangzhou, Guangdong, China

'Department of Operation Theater Services, Sun Yat-Sen University Cancer Center, State Key Laboratory of Oncology in South China, Collaborative Innovation Center of Cancer Medicine, Guangzhou, Guangdong, China

${ }^{\mathrm{d}}$ These authors contributed equally to this work

${ }^{e}$ Corresponding Author: Zhen Liao, Department of Operation Theater Services, Sun Yat-Sen University Cancer Center, State Key Laboratory of Oncology in South China, Collaborative Innovation Center of Cancer Medicine, 651 Dong Feng Dong Road, Guangzhou, Guangdong, 510060, China.

Email: liaozhen@sysucc.org.cn

doi: https://doi.org/10.14740/wjon1143w usually is found in the skin that occurs on the sun-exposed areas of the body [1]. The location of the lesion is most commonly confined to the scalp, forehead and neck, trunk, or upper extremities; and the TC generally has an indolent clinical course. This condition is usually considered curable after a wide excision [2]. Besides, the clinical feature is usually not taken seriously, with a recent onset of abruptly growth phase, which prompts medical evaluation in the late of disease progression [3]. Investigations of TC are rare, the tumor behavior and prognosis are difficult to predict [4]. Although TC has a low metastatic potential and it does not usually recur, any long-term follow-up data has not been collected on the patients with this neoplasm; and they can invade deeply and may recur locally [5]. In addition, there is no consensus on the optimal treatment for TC with metastasis and no established chemotherapy regimen for metastatic TC reported in the medical literature. Therefore, TC patients had a bad prognosis when metastasis occurred [6]. The risk of recurrence and lymph node metastasis may be probably similar to that of squamous cell carcinomas, but few data support such a contention [4].

However, most of the reported documents in the old literature are case reports; and very few studies have investigated the treatment strategies for this unique population. As we know, a large-scale study about TC has not been reported. Herein, to investigate this aspect, we performed a retrospective analysis of data obtained from the Sun Yat-Sen University Cancer Center. We evaluated survival rate, clinical features and prognostic factors in 26 patients who developed TC. We aimed to identify the optimal therapeutic strategy for TC comprehensively.

\section{Patients and Methods}

\section{Patient data}

This retrospective study enrolled consecutive patients who were admitted to the Sun Yat-Sen University Cancer Center between February, 1998 and February, 2012 with diagnoses of TC. Prior consent was obtained from patients, as was the approval of the Institutional Research Ethics Committee of the Sun Yat-sen University Cancer Center. In total 26 TC patients were eligible for our study (12 men and 14 women). The male to female ratio was 0.85 . The average age of patients with $\mathrm{TC}$ was 42.5 years.

Cumulative risk of all TC patients was estimated by using the Kaplan-Meier method. Cox proportional-hazard regression model was used to estimate the relative risk of survival after treatment. The difference was considered statistically signifi- 


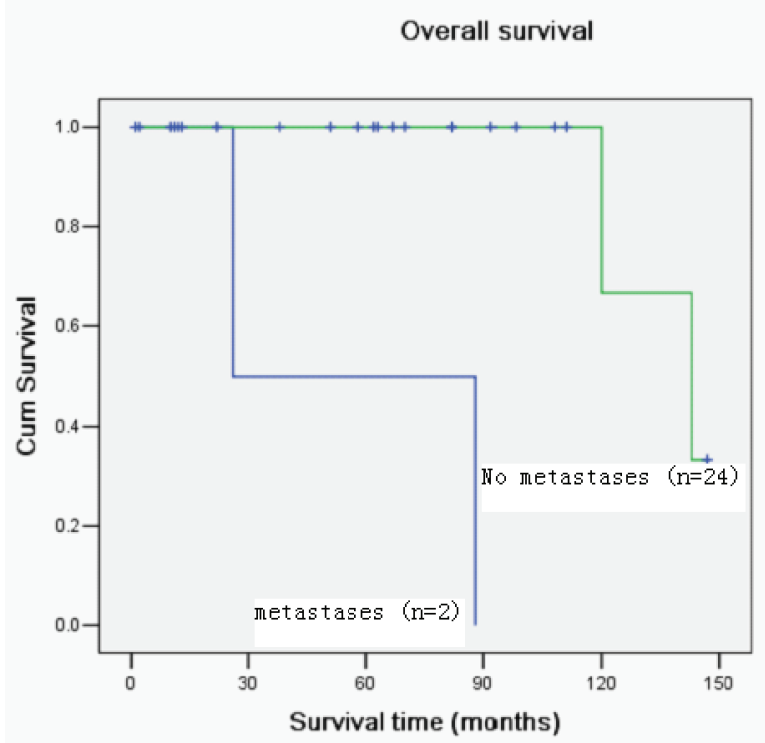

Figure 1. Kaplan-Meier curve with univariate analysis (log-rank) for patients with distance metastases versus no distance metastases for overall survival.

cant when the $\mathrm{P}$ value was less than 0.05 .

\section{Clinical data}

In our study data, most of the TC patients had a history of significant sun exposure throughout their lives. The pathological diagnosis of all patients was confirmed to be TC. Twelve of 26 patients were first treated elsewhere and presented to the reporting center at the time of the local recurrence development. The most common tumors sites in the patients were areas of sun-exposure, which included 14 cases located in occipitalia, six cases of scalp, two cases of temporal, two cases of ear, and napex and lower lip in one case, respectively. On macroscopic, the neoplasm had been described as exophytic, polypoid, ulcerated or nodular lesions, accompanied with or without malodorous and bleeding. The size of the tumors ranged from $1 \mathrm{~cm}$ to $8 \mathrm{~cm}$, with a mean size of $2.8 \mathrm{~cm}$. Twenty cases had clear borders, and six cases had unclear borders. In our data, in most of the TC patients the lesion had usually been presented for a long time before the diagnosis and had a recent rapid growth phase.

Follow-up data were collected from the outpatient service and complementary data were obtained by telephone inquiry and follow-up letters. The follow-up deadline was set as February 29,2012 . The median follow-up period for all patients was 63.8 months (range: 1 - 147 months).

\section{Treatment}

All of the patients were treated with surgery simply, complete surgical excision with documented clear margins intraoperative and postoperative were undertake for all patients: 11

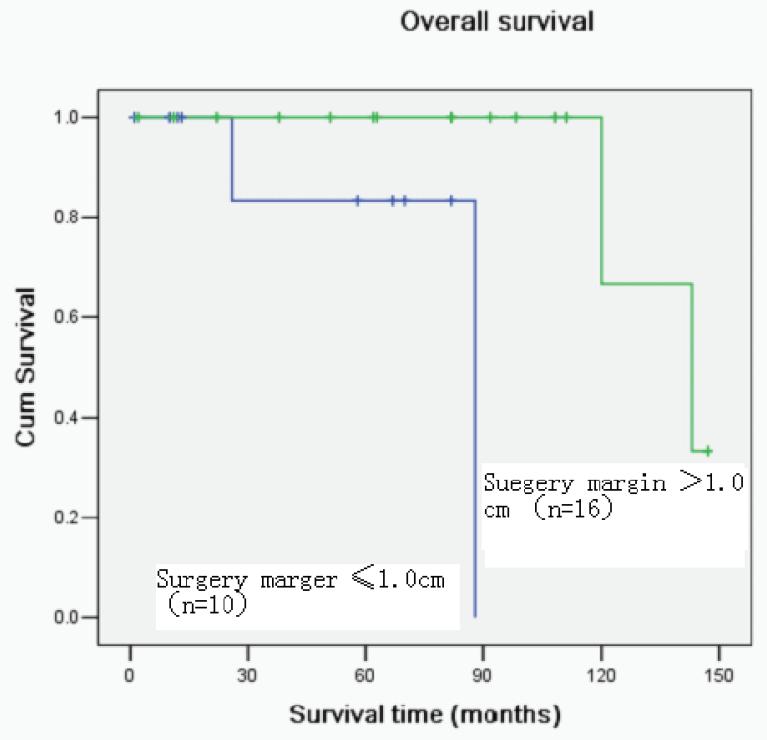

Figure 2. Kaplan-Meier curve with univariate analysis (log-rank) for patients treated with surgery margin $\leq 1.5 \mathrm{~cm}$ versus $>1.5 \mathrm{~cm}$ for overall survival.

patients to pull direct suture after surgery, in five patients reconstruction with rotation flap was performed; and the remaining 10 patients underwent local rotation flap reconstruction and skin-grafting. Split skin was obtained from the abdomen (seven cases) and anterolateral (three cases), meshed 1:1.5, and transplanted onto the dermal template. There was an uneventful postoperative course for all patients. But two patients developed lymph nodes metastasis and far recurrence after initial surgery, in whom the expand resection and neck dissection were taken. Chemotherapy (cisplatin and vindesine) treatment was given after the surgery.

\section{Result}

Of the 26 patients, four patients died. The overall 5-year survival rates of all patients were $89.2 \%$.Univariate analysis revealed that the key prognostic factors influencing the survival of these patients were the lymph nodes metastasis and surgery margin (Figs. 1, 2; Table 1). There was no significant correlation between TC and gender, tumor size, age and local recurrence.

In the Cox proportional hazard regression model, there was no risk factor influencing the survival of TC patients.

\section{Discussion}

TC is a rare skin adnexal malignant tumor deriving from the outer root sheath of hair follicles; it usually occurs on the sunexposed areas of the elderly [1,7]. TC is usually a solitary indolent lesion, although it can appear as multiple lesions on non-sun-exposed skin [8]. Clinically, it may be mistaken for squamous cell carcinoma, basal cell carcinoma, nodular mela- 
Table 1. Influence of Clinicopathologic Characteristics on the Survival of 26 Trichilemmal Carcinoma Patients by Log-Rank Test

\begin{tabular}{|c|c|c|c|c|}
\hline Characteristic & Cases & 5-year survival & $\chi^{2}$ value & P value \\
\hline Gender & & & 0.253 & 0.615 \\
\hline Male & 12 & 88.9 & & \\
\hline Female & 14 & 88.9 & & \\
\hline Age & & & 0.031 & 0.859 \\
\hline$>40$ years & 15 & 90.9 & & \\
\hline$\leq 40$ years & 11 & 87.5 & & \\
\hline Tumor size & & & 0.027 & 0.869 \\
\hline $\mathrm{D} \leq 2.5 \mathrm{~cm}$ & 12 & 88.9 & & \\
\hline $\mathrm{D}>2.5 \mathrm{~cm}$ & 14 & 90.0 & & \\
\hline $\mathrm{N}$ classification & & & 22.539 & 0.000 \\
\hline $\mathrm{N}+$ & 2 & 00.0 & & \\
\hline No & 24 & 87.5 & & \\
\hline Local recurrence & & & 2.793 & 0.095 \\
\hline Yes & 12 & 77.8 & & \\
\hline No & 14 & 100 & & \\
\hline Surgery margin & & & 8.131 & 0.004 \\
\hline$\leq 1.0 \mathrm{~cm}$ & 10 & 71.4 & & \\
\hline$>1.0 \mathrm{~cm}$ & 16 & 100 & & \\
\hline
\end{tabular}

noma, or keratoacanthoma. It has been shown to be a malignant form of a trichilemmoma [9]. The diagnosis is established by means of histopathological examination using hematoxylin-eosin staining which, when necessary, is complemented by immunohistochemistry of the lesions [10].

TC generally has a nonaggressive course. Boscaino and colleagues reported that, after excision of the lesions in seven patients, there was no recurrence of the tumor within a 2-month to 4-year follow-up [7]. The clinical findings in ours series were quite similar to those described by previous reports $[1,3$, $5,7,8-10]$. First, the duration of the lesion prior to diagnosis ranged from 2 months to 50 years. Rapid growth of tumor prior to presentation for a long time has been noted in some cases; clinically, these tumors have frequently been misdiagnosed as benign lesions. Besides, most of the TC patients had a history of significant sun exposure throughout their lives. The distribution of the lesions tends to suggest that sunlight may play an important role in the development of this malignancy [11]. However, TC is an adnexal tumor; its lesion distribution may simply reflect its appendageal origin which favors the head and neck region. Moreover, TC has been reported in burn scars, as well as in an elderly man who received between 50 and 60 diagnostic chest radiographs for management of pulmonary tuberculosis $[12,13]$. But the pathogenesis of TC remains poorly understood.

AS we know that TC is an unusual malignant lesion with the histological features suggesting an intermediate to high grade malignancy; and it generally has a benign neoplasm process and can be treated with complete excision [9]. Standard care for this tumor has been surgical excision with demonstration of clear margins [14]. Histologically clear margins are crucial for locally aggressive growth pattern and local recurrence potential $[7,9,14]$. In our data, despite the wide local excision with tumor-free margins, tumor recurrence occurred in two cases, but 24 of 26 patients were excised surgically with a satisfactory outcome. Surgery is considered the treatment of choice for TC, and periodic surveillance without adjuvant therapy is generally sufficient [12].

Local cervical lymph node metastases and distance metastases of this neoplasm have seldom been reported in the medical literature $[3,9,14]$. In our study, totally two patients died from involved regional lymph nodes, and presented with distance metastases. They had died in 1 year and 6 months later after surgery and chemotherapy (cisplatin and vindesine). Unfortunately, there is no consensus on the optimal treatment for TC with metastases. There is also no established chemotherapy regimen for metastatic TC reported in the medical literature. Yi HS et al reported that a patient was treated with four cycles of cisplatin and cyclophosphamide combination chemotherapy, and a partial remission was achieved. The cisplatin and cyclophosphamide treatment was not curative, but rather, it controlled the tumor growth [6]. Roismann $\mathrm{M}$ et al prescribed a case for four cycles of chemotherapy (5-fluouracil associated with cisplatin) with poor prognosis [10]. Moreover, we found that Hayashi et al administered chemotherapy (cisplatin, adriamycin, vindesine : CAV treatment) similar to the regimen used for highly advanced cases of squamous cell carcinoma $[15,16]$. The present study shows a recurrent TC case of difficult treatment, which presented distance metastases [4, $6,10,15,16]$.

In summary, we have reviewed the clinicopathologic findings in 26 patients of TC. The treatment is exclusively surgical. Simple excision with adequate margins is safe, inexpensive and effective for the treatment of TC; and postoperative follow-up of the patient to facilitate early diagnosis of the recurrence and distant metastasis is necessary. If tumor distance metastasis is confirmed, then systemic chemotherapy should be considered (four cycles of cisplatin and vindesine or four cycles of cisplatin and cyclophosphamide). It may control the disease progression.

\section{Grant Support}

None.

\section{References}

1. Reis JP, Tellechea O, Cunha MF, Baptista AP. Trichilemmal carcinoma: review of 8 cases. J Cutan Pathol. 1993;20(1):44-49.

2. Maize JC, Snider RL. Nonmelanoma skin cancers in association with seborrheic keratoses. Clinicopathologic correlations. Dermatol Surg. 1995;21(11):960-962.

3. Swanson PE, Marrogi AJ, Williams DJ, Cherwitz DL, Wick MR. Tricholemmal carcinoma: clinicopathologic study of 10 cases. J Cutan Pathol. 1992;19(2):100-109. 
4. Van Zele D, Arrese JE, Heymans O, Fissette J, Pierard GE. Invasive tricholemmal carcinoma of the nose. Dermatology. 2002;204(4):315-317.

5. Dailey JR, Helm KF, Goldberg SH. Tricholemmal carcinoma of the eyelid. Am J Ophthalmol. 1993;115(1):118119.

6. Yi HS, Sym SJ, Park J, Cho EK, Ha SY, Shin DB, Lee JH. Recurrent and metastatic trichilemmal carcinoma of the skin over the thigh: a case report. Cancer Res Treat. 2010;42(3):176-179.

7. Boscaino A, Terracciano LM, Donofrio V, Ferrara G, De Rosa G. Tricholemmal carcinoma: a study of seven cases. J Cutan Pathol. 1992;19(2):94-99.

8. Garrett AB, Scott KA. Trichilemmal carcinoma: a case report of a rare skin cancer occurring in a renal transplant patient. Transplantation. 2003;76(7):1131.

9. Garrett AB, Azmi FH, Ogburia KS. Trichilemmal carcinoma: a rare cutaneous malignancy: a report of two cases. Dermatol Surg. 2004;30(1):113-115.

10. Roismann M, Freitas RR, Ribeiro LC, Montenegro MF, Biasi LJ, Jung JE. Trichilemmal carcinoma: case report.
An Bras Dermatol. 2011;86(5):991-994.

11. Laochumroonvorapong P, Kokta V, Quan MB. Trichilemmal carcinoma in an African American. Dermatol Surg. 2002;28(3):284-286.

12. Ko T, Tada H, Hatoko M, Muramatsu T, Shirai T. Trichilemmal carcinoma developing in a burn scar: a report of two cases. J Dermatol. 1996;23(7):463-468.

13. Chan KO, Lim IJ, Baladas HG, Tan WT. Multiple tumour presentation of trichilemmal carcinoma. Br J Plast Surg. 1999;52(8):665-667.

14. Wong TY, Suster S. Tricholemmal carcinoma. A clinicopathologic study of 13 cases. Am J Dermatopathol. 1994;16(5):463-473.

15. Hayashi I, Harada T, Muraoka M, Ishii M. Malignant proliferating trichilemmal tumour and CAV (cisplatin, adriamycin, vindesine) treatment. Br J Dermatol. 2004;150(1):156-157.

16. Ikegawa S, Saida T, Obayashi H, Sasaki A, Esumi H, Ikeda S, Kiyohara Y, et al. Cisplatin combination chemotherapy in squamous cell carcinoma and adenoid cystic carcinoma of the skin. J Dermatol. 1989;16(3):227-230. 